\title{
Correlation between NADPH oxidase-mediated oxidative stress and dysfunction of endothelial progenitor cell in hyperlipidemic patients
}

\author{
Ting-Bo Li ${ }^{1,2,}{ }^{*}$, Yin-Zhuang Zhang, ${ }^{3,}$, Wei-Qi Liu ${ }^{3}$, Jie-Jie Zhang ${ }^{2}$, Jun Peng ${ }^{2,4}$, Xiu-Ju Luo ${ }^{1}$, and Qi-Lin Ma ${ }^{3}$
}

${ }^{1}$ Department of Laboratory Medicine, Xiangya School of Medicine, Changsha; ${ }^{2}$ Department of Pharmacology, School of Pharmaceutical Sciences, Central South University, Changsha; ${ }^{3}$ Department of Cardiovascular Medicine, Xiangya Hospital, Central South University, Changsha; ${ }^{4}$ Hunan Provincial Key Laboratory of Cardiovascular Research, School of Pharmaceutical Sciences, Central South University, Changsha, China

Received: April 23, 2016

Revised : July 19, 2016

Accepted: October 13, 2016

Correspondence to

Xiu-Ju Luo, Ph.D.

Department of Laboratory Medicine, Xiangya School of Medicine, Central South University, 172 Tong Zi Po Rd, Changsha 410013, China

Tel: +86-731-82650348

Fax: +86-731-82650348

E-mail:xjluo22@csu.edu.cn

* These authors contributed equally to this work.
Background/Aims: NADPH (nicotinamide adenine dinucleotide phosphate) oxidase (NOX)-mediated oxidative stress plays a key role in promotion of oxidative injury in the cardiovascular system. The aim of this study is to evaluate the status of NOX in endothelial progenitor cells (EPCs) of hyperlipidemic patients and to assess the correlation between NOX activity and the functions EPCs.

Methods: A total of 30 hyperlipidemic patients were enrolled for this study and 30 age-matched volunteers with normal level of plasma lipids served as controls. After the circulating EPCs were isolated, the EPC functions (migration, adhesion and tube formation) were evaluated and the status of NOX (expression and activity) was examined.

Results: Compared to the controls, hyperlipidemic patients showed an increase in plasma lipids and a reduction in EPC functions including the attenuated abilities in adhesion, migration and tube formation, concomitant with an increase in NOX expression (NOX2 and NOX4), NOX activity, and reactive oxygen species production. The data analysis showed negative correlations between NOX activity and EPC functions.

Conclusions: There is a positive correlation between the NOX-mediated oxidative stress and the dysfunctions of circulating EPCs in hyperlipidemic patients, and suppression of NOX might offer a novel strategy to improve EPCs functions in hyperlipidemia.

Keywords: NADPH oxidase; Hyperlipidemias; Endothelial progenitor cells; Reactive oxygen species; Oxidized low-density lipoprotein

\section{INTRODUCTION}

Hyperlipidemia, including hypercholesterolemia and hypertriglyceridemia, is considered a major risk factor for atherosclerosis and atherosclerosis-associated diseases such as coronary heart disease, stroke, and hypertension. Accumulating evidence suggests that increased plasma lipids, such as low density lipoprotein (LDL) and oxidized low density lipoprotein (ox-LDL) in particular, can result in vascular endothelial dysfunction and attenuate the repairment of lipoprotein-mediated endothelial injury via several mechanisms such as oxidative stress and inflammatory reaction [1-3]. Endothelial progenitor cells (EPCs), a heterogeneous cell population, are capable of differentiating into the endothelial lineage. EPCs are primarily derived from bone marrow (BM), but they can also be isolated from umbilical cord or peripheral blood. EPCs are thought to function as a backup system 
to repair endothelial injury so as to maintain vascular endothelial homeostasis [4-6]. Recent studies show that circulating EPCs contribute to the repairmen of endothelial injury in hypertension associated with hypercholesterolemia [7]. There is evidence that reduced numbers and impaired functional capacity of EPCs are found in cell culture with ox-LDL or in patients with hypercholesterolemia $[8,9]$. Under such conditions, the functions of EPCs in maintaining endothelial integrity and vascular homeostasis are compromised. It is not known, however, what are the potential mechanisms responsible for dysfunction of EPCs under the condition of hyperlipidemia.

Oxidative stress occurs when there is an increased production of reactive oxygen species (ROS) and/or a significant decrease in antioxidant enzyme activity. It has been shown that ROS plays critical roles in regulating stem and progenitor cell bioactivity and that oxidative stress not only reduces EPCs numbers but also impairs EPCs functions (such as migration, adhesion and neovascularization) in various pathological situations [10-12]. Of the multiple sources of ROS in the blood vessel, nicotinamide adenine dinucleotide phosphate (NADPH) oxidase (NOX) is the primary one $[5,13]$. There are reports that over production of NOX-derived ROS not only hurts the endothelial cells but also causes EPCs dysfunctions [5,14,15]. In hypercholesterolemic mice, administration of NOX inhibitor (apocynin) reduces ROS production and improves the functions of endothelial cells $[11,16]$. The production of ROS is dramatically increased in patients with hyperlipidemia. It is unclear, however, whether NOX is also activated in EPCs from hyperlipidemic patients and whether the NOX-derived ROS is related to the functional impairment of EPCs.

The aims of this study were to explore the status of NOX in EPCs from hyperlipidemic patients and its association with EPCs functions. In this study, by collecting the EPCs from peripheral blood of the hyperlipidemic patients and the controls, we evaluated the correlation between NOX activity and EPCs functions.

\section{METHODS}

\section{Study subjects}

Patients with hyperlipidemia $(\mathrm{n}=30)$ and healthy vol- unteers $(n=30)$ from Xiangya Hospital (Changsha, China) were enrolled for this study. All patients were newly diagnosed with hyperlipidemia based on the results of total cholesterol (TC) and/or total triglycerides (TG) examinations. Exclusion criteria included cardiomyopathy, congenital heart disease, liver or renal diseases, bleeding disorders, and malignant diseases. Age- and sex-matched controls were selected from community-based inhabitants, free of any hyperlipidemia following the same exclusion criteria as mentioned above. The relevant characteristics of hyperlipidemic patients and controls are summarized in Table 1.

\section{Isolation and characterization of EPCs}

Peripheral venous blood samples ( $15 \mathrm{~mL}$ ) from the enrolled patients or controls were collected in a sterile tube containing the anticoagulant heparin for plasma or EPCs isolation. EPCs were isolated and cultured according to instructions provided by commercial kits. Briefly, EPCs were obtained by density gradient centrifugation with Ficoll-Paque PREMIUM (GE-Healthcare, Freiburg, Germany). After washing, $10^{7}$ cells were seeded on culture plates coated with human fibronectin (Millipore, Temecula, CA, USA) and maintained in endothelial basal medium-2 (Lonza, Walkersville, MD, USA) containing endothelial growth medium-2 (EGM-2) SingleQuots (Lonza). At the 7 th day of culture, EPCs were identified by immunofluorescence staining to detect surface markers and dual binding characteristics of acetylated LDL incorporation and lectin.

\section{Measurement of plasma lipids}

For the measurement of the levels of total TC, TG, LDL and high density lipoprotein in plasma, commercial diagnostic kits were used and the measurements were carried out according to the instructions provided by the manufacturer (Jiancheng Institute of Biotechnology, Nanjing, China). The levels of ox-LDL in plasma were assayed using a commercial ELISA (enzyme-linked immunosorbent assay) kit (R\&D Systems, Minneapolis, MN, USA).

\section{Immunofluorescence staining}

EPCs were verified by immunofluorescence staining with primary antibody against $\mathrm{CD}_{34}$ (Santa Cruz Biotechnology, Santa Cruz, CA, USA), fetal liver kinase 1 
Table 1. Body weight and plasma lipids

\begin{tabular}{|c|c|c|c|}
\hline Variable & Control & Patient & $p$ value \\
\hline Total subject & 30 & 30 & \\
\hline Age, yr & $57 \cdot 3 \pm 1.7$ & $54 \cdot 4 \pm 1.1$ & 0.158 \\
\hline Male sex & $17(56.7)$ & $15(50.0)$ & 0.795 \\
\hline Body weight, kg & $62.3 \pm 1.8$ & $63.6 \pm 2.0$ & 0.631 \\
\hline Hypertension & $8(0.27)$ & $14(0.47)$ & 0.180 \\
\hline Diabetes & 0 & o & \\
\hline Smoking & $5(0.17)$ & $8(0.27)$ & 0.531 \\
\hline $\mathrm{TC}, \mathrm{mmol} / \mathrm{L}$ & $4.00 \pm 0.18$ & $6.85 \pm 0.27$ & $<0.010$ \\
\hline $\mathrm{TG}, \mathrm{mmol} / \mathrm{L}$ & $1.35 \pm 0.12$ & $2.48 \pm 0.40$ & $<0.010$ \\
\hline $\mathrm{LDL}, \mathrm{mmol} / \mathrm{L}$ & $2.26 \pm 0.10$ & $4.29 \pm 0.18$ & $<0.010$ \\
\hline ox-LDL, $\mu \mathrm{g} / \mathrm{L}$ & $109.80 \pm 10.58$ & $164.30 \pm 13.87$ & $<0.010$ \\
\hline $\mathrm{HDL}, \mathrm{mmol} / \mathrm{L}$ & $1.21 \pm 0.04$ & $1.72 \pm 0.09$ & $<0.050$ \\
\hline
\end{tabular}

Values are presented as mean \pm SD or number (\%).

TC, total cholesterol; TG, total triglycerides; LDL, low density lipoprotein; ox-LDL, oxidized low density lipoprotein; HDL, high density lipoprotein.

(Flk-1, Abcam, Cambridge, MA, USA), CD144 (Santa Cruz Biotechnology), CD31 (Abcam), endothelial nitric oxide synthase (eNOS, Life Science, Shanghai, China), followed by a secondary antibody of goat anti-rabbit Cy3-conjugated immunoglobulin G (IgG, Beyotime, Jiangsu, China) or goat anti-mouse DyLight 488-conjugated IgG (Beyotime). The cell nuclei were labeled with 4,6-diamidino-2-phenylindole (DAPI, Beyotime). Images were taken under a fluorescence microscope (Olympus IX 71 , Olympus, Tokyo, Japan). For the negative control, PBS was utilized to replace the primary antibodies.

\section{Functional analysis of EPCs}

The functions of EPCs were assessed through evaluating the abilities of migration, adhesion and tube formation. For migration assay, $1 \times 10^{5} \mathrm{EPCs}$ were put in the upper chamber of a 24-well Transwell (Corning, NY, USA), and $600 \mu \mathrm{L}$ EGM-2 media containing $50 \mathrm{ng} / \mathrm{mL}$ of vascular endothelial growth factor were placed to the lower compartment of the chamber. After 6 hours incubation at $37^{\circ} \mathrm{C}$, media in the upper and lower chamber were aspirated and EPCs on the top membrane were gently wiped off with a cotton swab. The Transwell membrane was washed with PBS and then fixed in $4 \%$ paraformaldehyde for 15 minutes at room temperature. For quantitation, $0.1 \%$ crystal violet solution (Beyotime) was used to stain cells, and cells migrating into the lower chamber were counted manually in ten random fields under $\times 200$ magnification.

For adhesion assay, $5 \times 10^{4} \mathrm{EPCs}$ were seeded in 12-well plates coated with $10 \mu \mathrm{g} / \mathrm{mL}$ human plasma fibronectin (Millipore). After 30 minutes incubation, plates were washed three times with PBS to remove non-adherent cells, and then incubated in $4 \%$ paraformaldehyde. Cells were stained with DAPI (Beyotime) and counted in five random fields under $\times 100$ magnification.

For tube formation assay, Matrigel basement membrane matrix (ECMatriX, BD Biosciences, Franklin Lakes, NJ, USA) was thawed at $4^{\circ} \mathrm{C}$ overnight. Then 60 $\mu \mathrm{L}$ matrigel was added in a 96-well plate for 1 hour at $37^{\circ} \mathrm{C}$. Ten thousand EPCs were added on the top of the solidified matrix containing EGM-2. After 16 hours incubation, tube morphology was imaged and 10 random fields were counted for the tube numbers under $\times 40$ magnification.

\section{Measurement of ROS production in EPCs}

Intracellular ROS levels were measured based on the fluorescent signal of 2',7'-dichlorodihydrofluorescein diacetate (DCFH-DA), a cell-permeable ROS indicator (Beyotime). Non-fluorescent DCFH-DA is oxidized to fluorescent DCF by intracellular ROS once the acetate groups are removed. Briefly, EPCs were washed with PBS and then incubated with $10 \mu \mathrm{M}$ DCFH-DA at $37^{\circ} \mathrm{C}$ 
Table 2. Primers for real-time polymerase chain reaction

\begin{tabular}{llll}
\hline Gene & \multicolumn{1}{c}{ Forward primer } & \multicolumn{1}{c}{ Reverse primer } & Product size, bp \\
\hline NOX 2 & GGGAACTGGGCTGTGAATGA & CCAGTGCTGACCCAAGAAGT & 148 \\
NOX $_{4}$ & AATGCACCAACAAATGGGGC & ACCTATTCGACACTGGAGATGC & 170 \\
$\beta$-Actin & TGACGTGGACATCCGCAAAG & CTGGAAGGTGGACAGCGAGG & 162 \\
\hline
\end{tabular}

NOX, NADPH (nicotinamide adenine dinucleotide phosphate) oxidase.

for 20 minutes. Then, the ROS-mediated fluorescence was monitored under a fluorescent microscope with excitation at $502 \mathrm{~nm}$ and emission at $523 \mathrm{~nm}$. Arbitrary fluorescent units were normalized to the control and presented as fold changes.

\section{Measurement of $\mathrm{H}_{2} \mathrm{O}_{2}$ production in EPCs}

To measure the $\mathrm{H}_{2} \mathrm{O}_{2}$ generation in EPCs, mixtures of 50 $\mu \mathrm{L}$ of EPCs lysate and $100 \mu \mathrm{L}$ of work solution $(0.25 \mathrm{mM}$ ammonium ferrous II sulfate, $100 \mathrm{mM}$ sorbitol, $25 \mathrm{mM}$ $\mathrm{H}_{2} \mathrm{SO}_{4}$, and $125 \mathrm{mM}$ xylenol orange) were incubated at room temperature for 20 minutes. The change of absorbance at $560 \mathrm{~nm}$ was observed, and the level of $\mathrm{H}_{2} \mathrm{O}_{2}$ was computed according to a standard curve made from the standard solutions offered by the supplier (Beyotime).

\section{Measurement of NOX activity}

The NOX activities were measured by analyzing the NADPH-dependent superoxide dismutase (SOD)-inhibited cytochrome c reduction (Genmed, Shanghai, China). Briefly, the reaction buffer $(900 \mu \mathrm{L})$ with the NOX substrate (NADPH) and the oxidized cytochrome $\mathrm{c}$ was incubated in a quartz cuvette at $30^{\circ} \mathrm{C}$ for 3 minutes, and then an aliquot $(100 \mu \mathrm{L})$ of the supernatant from EPCs lysate was incubated with the reaction mixture at $30^{\circ} \mathrm{C}$ for 15 minutes. The change of absorbance at $550 \mathrm{~nm}$ was detected in a spectrophotometer. SOD-inhibited cytochrome c reduction in buffer blank was monitored and subtracted from each sample. The NOX activity was evaluated as SOD-inhibited cytochrome c reduction and expressed as $\mathrm{O}^{2-}$ in $\mathrm{nmol} / \mathrm{mg} / \mathrm{min}$.

\section{Real-time polymerase chain reaction}

Real-time polymerase chain reaction (PCR) was performed to determine mRNA levels of $\mathrm{NOX}_{2}$ and $\mathrm{NOX}_{4}$ in EPCs, and $\beta$-actin served as a loading control. The primers for $\mathrm{NOX}_{2}, \mathrm{NOX}_{4}$, and $\beta$-actin were listed in Ta- ble 2. Real-time PCR was conducted following standard protocols. Briefly, $2 \mu \mathrm{g}$ of total RNA from each sample was subjected to reverse transcription reaction by Reverse Transcription System (Takara, Kyoto, Japan) to obtain the cDNAs. Then, $10 \mu \mathrm{L}$ of reaction mixtures containing $5 \mu \mathrm{L}$ SYBR Master mix, $2 \mu \mathrm{L}$ cDNA template, $2.4 \mu \mathrm{L} \mathrm{H}_{2} \mathrm{O}, 0.20 \mu \mathrm{L}$ ROX, and $0.20 \mu \mathrm{L}$ of each primer were amplified following the procedures: denaturing at $95^{\circ} \mathrm{C}$ for 10 minutes and 45 cycles of the amplification steps (denaturation at $95^{\circ} \mathrm{C}$ for 15 seconds, annealing and extension at $60^{\circ} \mathrm{C}$ for 1 minute). Data analysis was carried out by comparative cycle threshold method using 7300 System SDS Software (Applied Biosystems, Foster City, CA, USA). Results were expressed as the ratio of NOX2 or NOX4 mRNA to $\beta$-actin mRNA.

\section{Western blot analysis}

EPCs were homogenized in ice-cool lysis buffer, sonicated for 1 minute, and then centrifuged at $15,000 \mathrm{xg}$ for 15 minutes. The protein concentration in supernatants was determined by a BCA Protein Assay kit (Beyotime). Samples containing $\sim 40 \mu \mathrm{g}$ of protein were loaded to 10\% SDS-PAGE (sodium dodecyl sulfate polyacrylamide gel electrophoresis) gel and then transferred to polyvinylidene fluoride membranes. The membranes were incubated with primary antibodies against $\mathrm{NOX}_{2}$, or $\mathrm{NOX}_{4}$, followed by horseradish peroxidase (HRP)-conjugated secondary antibodies. The signals of bands were examined using Luminata Crescendo Western HRP substrate through Molecular Imager ChemiDoc XRS System (Bio-Rad, Philadelphia, PA, USA). The densitometric quantification was performed with Image J 1.43 (NIH, Bethesda, MD, USA; https://imagej.nih.gov/ij/). Antibody against $\beta$-actin (Beyotime) was used as loading control. 

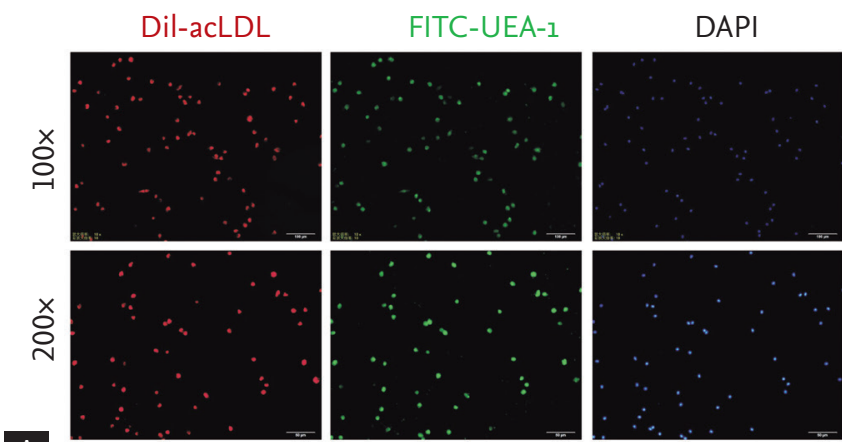

A

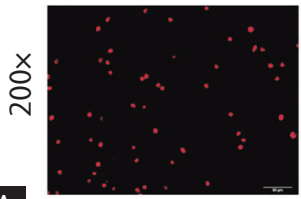

eNOS

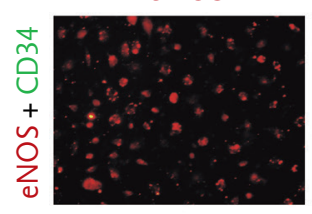

eNOS

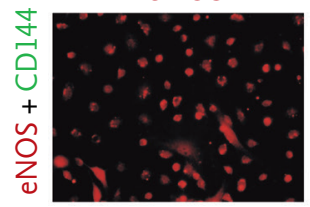

Flk-1

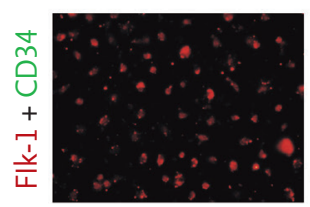

B

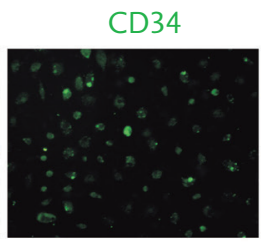

CD144

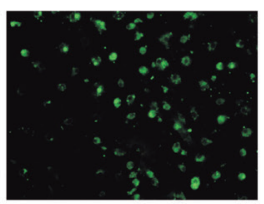

CD31
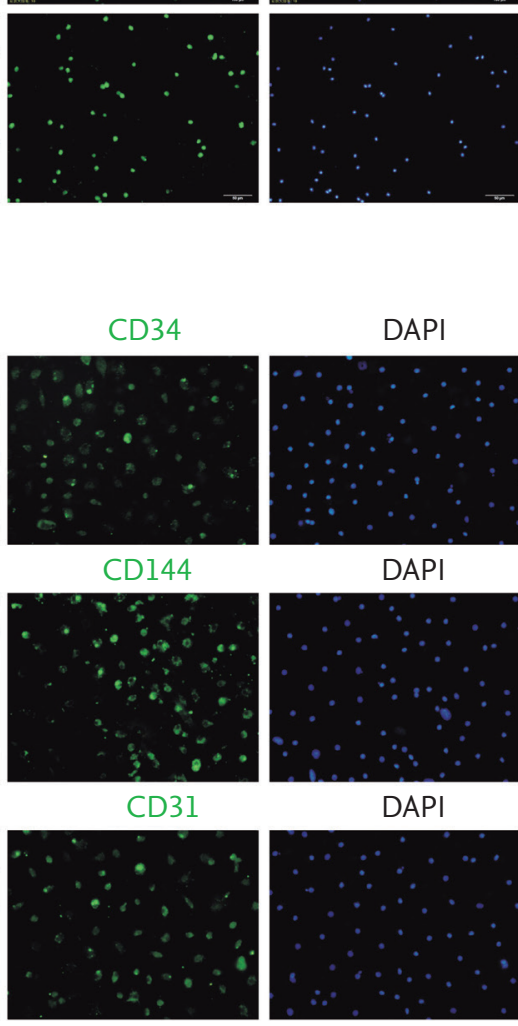

DAPI

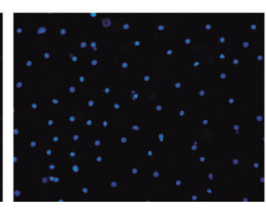

DAPI

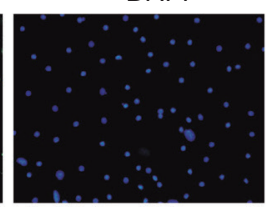

DAPI

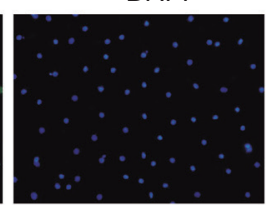

\section{Ethics}

This study has been performed in accordance with the Declaration of Helsinki (2013) of the World Medical Association, and was approved by the local Ethics Committee at Xiangya Hospital of Central South University (Changsha, China). Written informed consent was obtained from all individuals at the time of enrollment. All investigators were qualified to undertake this study.

\section{Statistics}

The statistical analysis was conducted using the SPSS version 20.0 (IBM Corp., Armonk, NY, USA). Data were presented as mean \pm standard error of the mean (SEM). Differences in measured values between two groups were assessed by Student $t$ test. For categorical variables, the chi-square test was used. The correlations between functions of EPCs and NOX activity were calculated us-
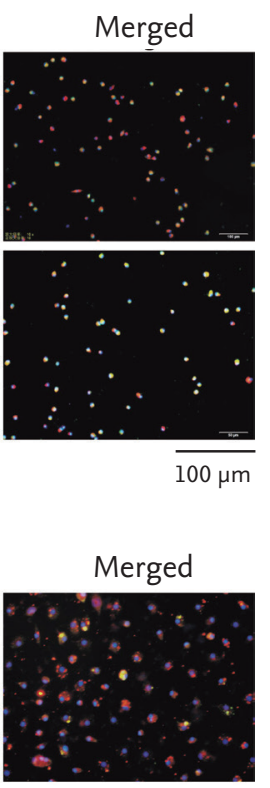

Merged

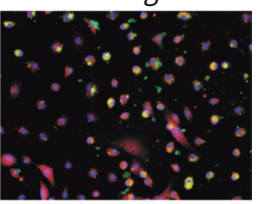

Merged

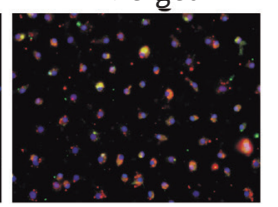

$\overline{100 \mu \mathrm{m}}$
Figure 1. Characterization of human endothelial progenitor cells (EPCs) from peripheral blood. (A) Three-color fluorescent imaging shows Dil-acetylated low density lipoprotein (Dil-acLDL) incorporation (red) and FITC lectin (FITC-UEA-1) binding (green) by EPCs. Nuclei appear dark blue (DAPI). (B) Immunofluorescence staining indicates the expression of $\mathrm{CD}_{34}$ (the stem cell marker), Flk-1, CD31, CD144, and eNOS (the endothelial cell markers) in EPCs. Nuclei were counterstained with DAPI (blue).

ing Pearson correlation coefficient. Differences were considered as significant when $p<0.05$.

\section{RESULTS}

\section{Phenotypic characterization of human EPCs}

The isolated mononuclear cells from human peripheral blood samples were cultured for 7 days. At the 7 th day, EPCs were identified as the attached cells, showing functions of acetylated LDL uptake (incorporation) and lectin binding (Fig. 1A), as well as expressing the stem cell marker (CD34) and endothelial cell markers (eNOS, CD144 Flk-1, and CD31) (Fig. 1B). 

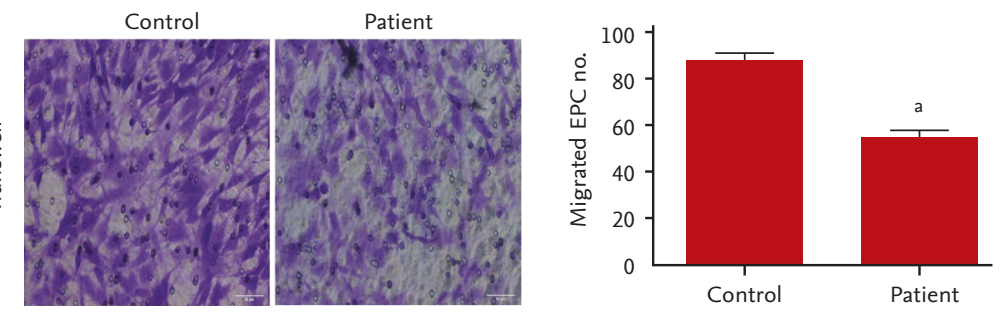

B
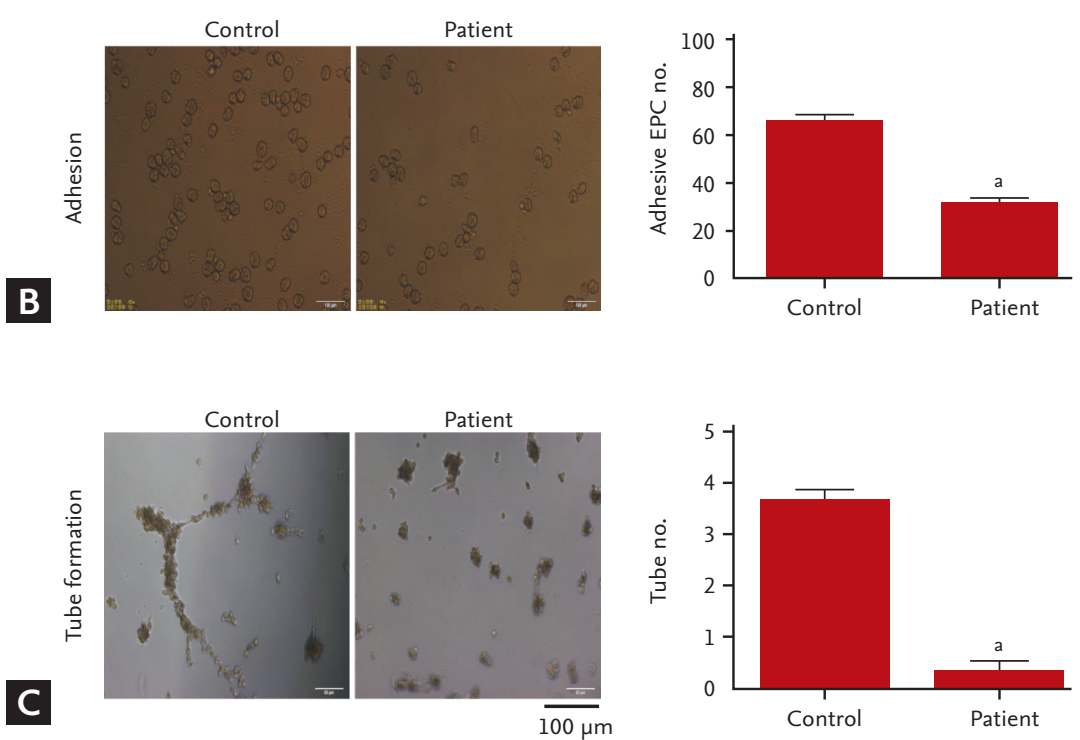

Figure 2. Dysfunctions of the circulating endothelial progenitor cells (EPCs) in hyperlipidemic patients. (A) Left panel, representative images for EPCs migration (Transwell) assay; right panel, the migrated EPC numbers. (B) Left panel, representative images for EPCs adhesion assay; right panel, the adhesive EPC numbers. (C) Left panel, representative images for EPCs tube formation assay; right panel, the tube numbers. All values are presented as mean \pm SEM (7 individual samples per group for migration, adhesion and tube formation analysis). ${ }^{a} p<0.01$ vs. control.

\section{Dysfunction of circulating EPCs in hyperlipidemic patients}

The functions of EPCs including the abilities of migration, adhesion, and tube formation were evaluated. Compared to the controls, EPCs migration was dramatically reduced in the hyperlipidemia patients (Fig. 2A), concomitant with decreases in adhesive EPCs numbers (Fig. 2B) and tube numbers (Fig. 2C).

\section{Up-regulation of NOX in EPCs from hyperlipidemic patients}

To investigate whether NOX, a major source of ROS in cardiovascular system, involved in the dysfunction of EPCs in hyperlipidemic patients, we measured the expression of NOX (NOX2 and NOX 4 ) and analyzed the correlation between NOX activity and EPC functions. Compared to the controls, mRNA and protein levels of NOX2 and $\mathrm{NOX}_{4}$ in the EPCs of the hyperlipidemic patients were significantly up-regulated (Fig. 3). Consistently, NOX activity was dramatically increased concomitant with an elevation in plasma levels of $\mathrm{H}_{2} \mathrm{O}_{2}$ and the intracellular ROS levels (Fig. 4). The correlation analysis showed that NOX activity in EPCs was inversely correlated with the EPC functions including the abilities of migration and adhesion (Fig. 5).

\section{DISCUSSION}

In the present study, we investigated the correlation between NOX and circulating EPCs functions in patients with hyperlipidemia. Our results showed that EPCs functions (migration, adhesion, and tube formation) were impaired in the patients with hyperlipidemia, accompanied by up-regulated NOX (NOX2 and NOX4) expressions, increased NOX activity, and NOX-derived products $\left(\mathrm{H}_{2} \mathrm{O}_{2}\right)$. The NOX activity was inversely correlated with the EPC functions (the abilities of migration and adhesion). To the best of our knowledge, this is the first study to provide evidence that NOX-mediatedoxidative stress is positively related to the dysfunctions of circulating EPCs in patients with hyperlipidemia.

Vascular endothelium plays a key role in regulation of vascular tone, structure, growth, and homeostasis, and 
A
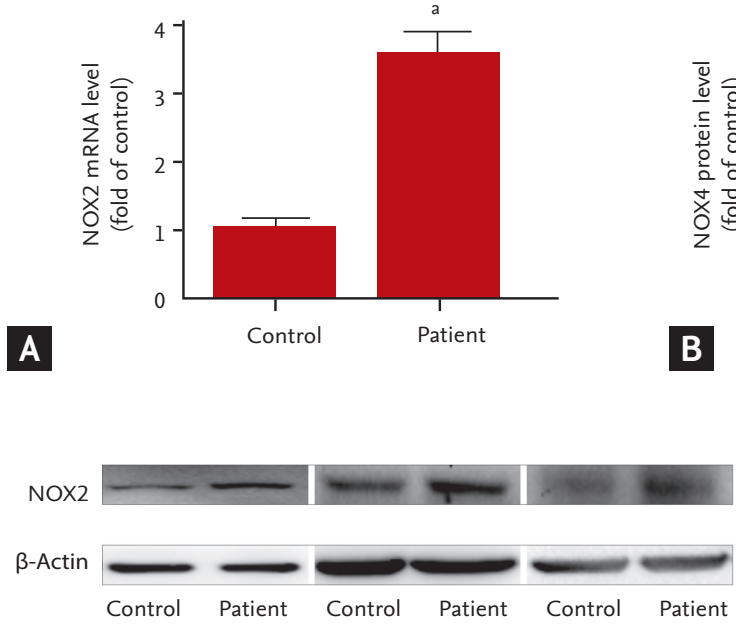

C

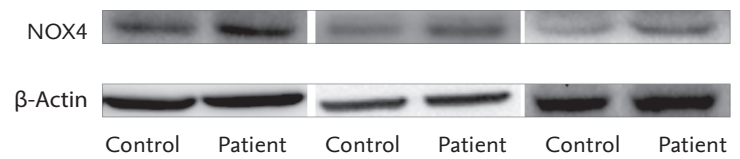

D
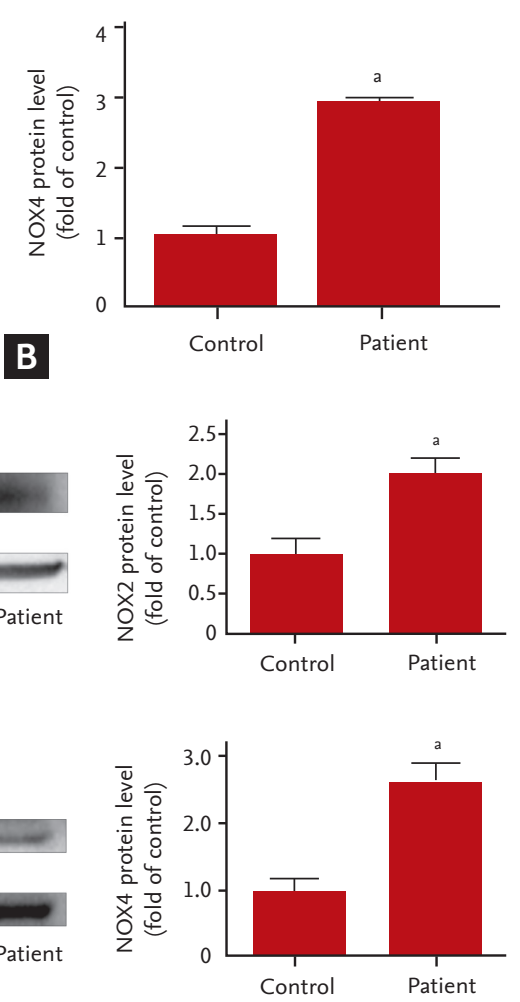

Figure 3. Up-regulation of $\mathrm{NOX}_{2}$ and $\mathrm{NOX}_{4}$ in endothelial progenitor cells (EPCs) of hyperlipidemic patients. (A, B) $\mathrm{NOX}_{2}$ or $\mathrm{NOX}_{4}$ mRNA expression. (C, D) NOX2 or $\mathrm{NOX}_{4}$ protein expression. Left, representative images of Western blot results for $\mathrm{NOX}_{2}$ or $\mathrm{NOX}_{4}$ and $\beta$-actin; right, relative changes in optical density for $\mathrm{NOX}_{2}$ or $\mathrm{NOX}_{4}$. All values are presented as mean \pm SEM (6 individual samples per group for mRNA and protein analysis, respectively). NOX, NADPH oxidase. ${ }^{\mathrm{a}} \mathrm{p}<0.01$ vs. control.
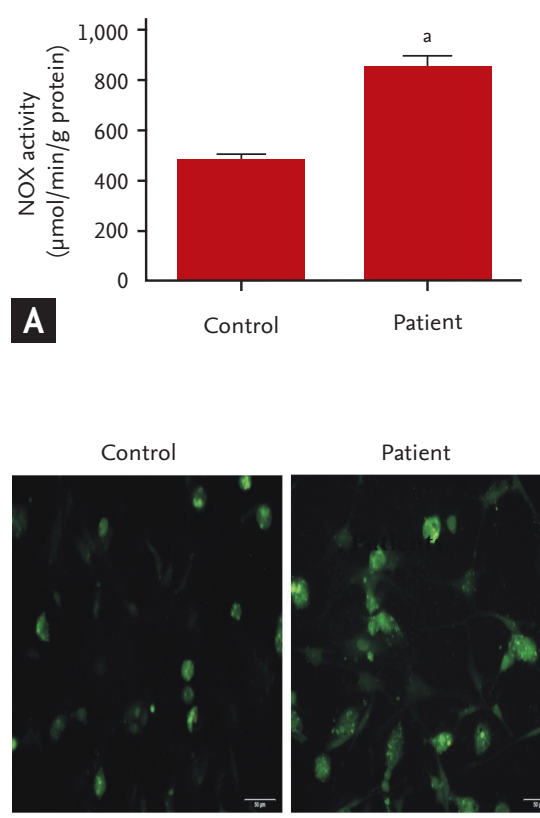

C

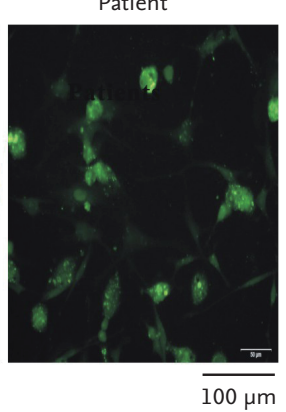

$\overline{100 \mu \mathrm{m}}$
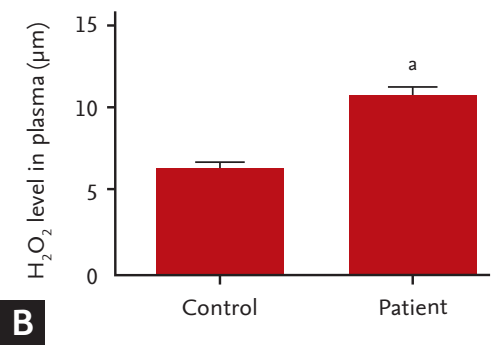

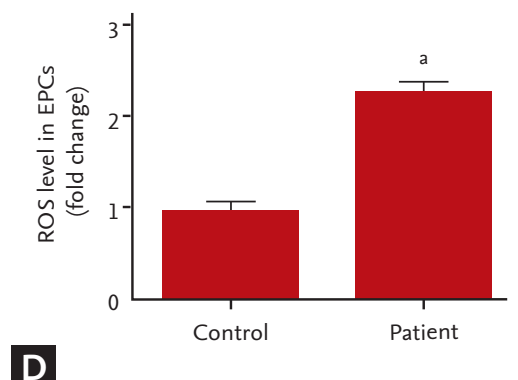

Figure 4. Increase of NADPH oxidase (NOX) activity and reactive oxygen species (ROS) production in endothelial progenitor cells (EPCs) of hyperlipidemic patients. (A) NOX activity in EPCs. (B) $\mathrm{H}_{2} \mathrm{O}_{2}$ levels in plasma. (C) Representative image of fluorescent signal of 2',7'-dichlorodihydrofluorescein diacetate (DCFHDA) for total ROS in EPCs. (D) Statistic value for fluorescent density. All values are presented as mean \pm SEM (7 individual samples per group for NOX activity and ROS level analysis). ${ }^{a} p<0.01$ vs. control. 

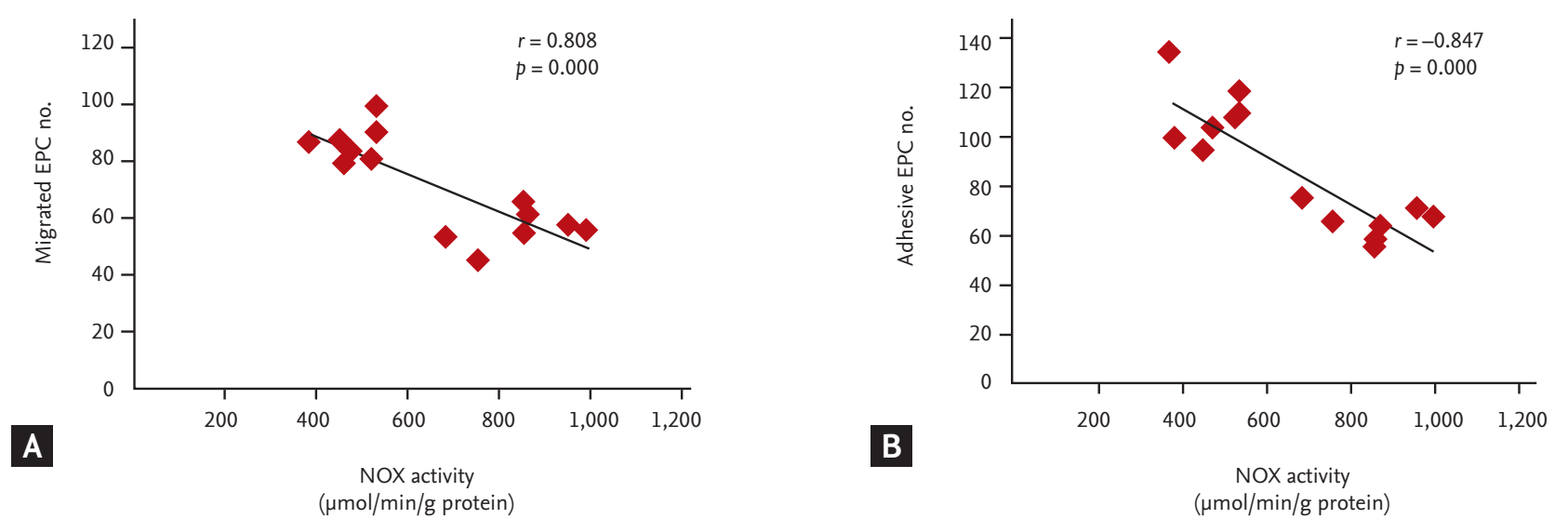

Figure 5. Negative correlation between NADPH oxidase (NOX) activity and endothelial progenitor cells (EPCs) migration (A) or adhesion (B).

thus protects the vessels from inflammation, immune reaction and thrombosis [17,18]. Multiple stimuli (such as hyperlipidemia and hyperglycemia) can damage the endothelium, which triggers the concomitant invasion of macrophages and lipid depositions, a critical step in atherosclerosis development [19]. Therefore, prompt recovery of the injured endothelium is important for preventing the progression of atherosclerotic vascular disease in patients at risk. EPCs, a cluster of precursor cells that express some cell surface markers of stem cells and endothelial cells, have been reported to contribute to the restoration of the endothelial monolayer [20]. EPCs can be mobilized from the BM, by various stimuli, into the peripheral blood and contribute to the neo-angiogenic process or to the repair of the damaged endothelial cells [17]. There is evidence that the EPCs functions are impaired in hyperlipidemic patients, which may contribute to, at least partly, the endothelial dysfunction in such patients. Consistent with these reports, we have also found the EPCs functions (migration, adhesion, and tube formation) were attenuated in the hyperlipidemic patients. However, the underlying mechanisms are not fully elucidated.

Accumulating evidence demonstrates that oxidative stress is involved in hyperlipidemia-induced endothelial injury and dysfunction. The levels of ROS are significantly elevated in hyperlipidemic patients. It is well known that ROS are produced from several differentially localized and expressed enzyme systems in- cluding NOX, xanthine oxidase and cytochrome p450. Among them, NOX is a major source of ROS by transferring electrons from NADPH to oxygen to generate superoxide anion, which in turn dismutates into $\mathrm{H}_{2} \mathrm{O}_{2}$ either spontaneously or catalyzed by SOD $[21,22]$. Reports from other labs and ours repeatedly demonstrated that NOX-derived ROS accounts for cellular oxidative injury under multiple pathological conditions such as ischemia, hyperglycemia and hypertension [23-28]. It is reasonable to predict that NOX-derived ROS may also involve in EPCs dysfunctions in hyperlipidemic patients. Our results clearly showed that the NOX activity in EPCs was dramatically elevated concomitant with an increase in $\mathrm{ROS}\left(\mathrm{H}_{2} \mathrm{O}_{2}\right)$ production. To evaluate the correlation between NOX activity and EPCs functions, we performed the correlation analysis. The results showed that NOX activity in EPCs was inversely correlated with EPCs functions (migration and adhesion). These results suggested that NOX-derived ROS might be associated with EPCs dysfunctions in hyperlipidemic patients.

NOX family comprises seven members, named NOX1 to $\mathrm{NOX}_{5}$, dual oxidase (Duox)-1 and Duox-2. Among these isoforms, NOX1, NOX2 (also named as gp91phox) and $\mathrm{NOX}_{4}$ are major isoforms expressed in cardiovascular systems; $\mathrm{NOX}_{3}$ is highly expressed in the cochlea; $\mathrm{NOX}_{5}$ mainly exists in lymphoid tissue and testis while Duox-1 and Duox-2 are predominantly expressed in thyroid epithelial cells [5]. Recently, we have demonstrated that $\mathrm{NOX}_{2}$ and $\mathrm{NOX}_{4}$ expressions in EPCs from pul- 
monary hypertensive rats are dramatically up-regulated [29]. There is no significant change in NOXı expression while the $\mathrm{NOX}_{3}$ mRNA expression is not detectable. We thus focused on $\mathrm{NOX}_{2}$ and $\mathrm{NOX}_{4}$ in the present study. Similarly, NOX2 and NOX 4 expressions were dramatically up-regulated in EPCs from the hyperlipidemic patients, indicating that selective inhibition of NOX subtypes such as $\mathrm{NOX}_{2}$ and/or $\mathrm{NOX}_{4}$ may provide a strategy to prevent EPCs dysfunctions in patients with hyperlipidemia. However, the mechanisms responsible for the up-regulation of $\mathrm{NOX}_{2}$ and $\mathrm{NOX}_{4}$ in hyperlipidemia need to be further investigated.

The results presented in this study demonstrate, for the first time, that NOX was up-regulated in EPCs from the hyperlipidemic patients, and NOX-derived ROS was positively correlated with the EPCs dysfunctions. Thus, increased NOX might be a novel risk factor for EPCs dysfunctions in hyperlipidemia, and targeting NOX, particularly NOX2 and/or NOX4, may have clinical potential in treating endothelial dysfunction-related diseases.

\section{KEY MESSAGE}

1. Hyperlipidemic patients show a reduction in endothelial progenitor cell (EPC) functions (migration, adhesion, and tube formation).

2. NADPH (nicotinamide adenine dinucleotide phosphate) oxidase (NOX) is up-regulated in EPCs from the hyperlipidemic patients.

3. NOX-derived reactive oxygen species is positively correlated with the EPCs dysfunctions.

\section{Conflict of interest}

No potential conflict of interest relevant to this article was reported.

\section{Acknowledgments}

This work was supported by National Natural Science Foundation of China (No.81370250 to Qi-Lin Ma; No. 81573430 to Xiu-Ju Luo; No.81373409 to Jun Peng), Natural Science Foundation of Hunan Province, China (No.13JJ2008 to Jun Peng; No.2015JJ2156 to Luo XJ).

\section{REFERENCES}

1. Hermida N, Balligand JL. Low-density lipoproteincholesterol-induced endothelial dysfunction and oxidative stress: the role of statins. Antioxid Redox Signal 2014;20:1216-1237.

2. Liu YH, Liu Y, Chen JY, et al. LDL cholesterol as a novel risk factor for contrast-induced acute kidney injury in patients undergoing percutaneous coronary intervention. Atherosclerosis 2014;237:453-459.

3. Messner B, Bernhard D. Smoking and cardiovascular disease: mechanisms of endothelial dysfunction and early atherogenesis. Arterioscler Thromb Vasc Biol 2014;34:509515.

4. George AL, Bangalore-Prakash P, Rajoria S, et al. Endothelial progenitor cell biology in disease and tissue regeneration. J Hematol Oncol 2011;4:24.

5. Peng J, Liu B, Ma QL, Luo XJ. Dysfunctional endothelial progenitor cells in cardiovascular diseases: role of NADPH oxidase. J Cardiovasc Pharmacol 2015;65:80-87.

6. Urbich C, Dimmeler S. Endothelial progenitor cells: characterization and role in vascular biology. Circ Res 2004;95:343-353.

7. Alexandru N, Popov D, Dragan E, Andrei E, Georgescu A. Circulating endothelial progenitor cell and platelet microparticle impact on platelet activation in hypertension associated with hypercholesterolemia. PLoS One 2013;8:e52058.

8. Imanishi T, Hano T, Matsuo Y, Nishio I. Oxidized lowdensity lipoprotein inhibits vascular endothelial growth factor-induced endothelial progenitor cell differentiation. Clin Exp Pharmacol Physiol 2003;30:665-670.

9. Rossi F, Bertone C, Michelon E, Bianco MJ, Santiemma V. High-density lipoprotein cholesterol affects early endothelial progenitor cell number and endothelial function in obese women. Obesity (Silver Spring) 2013;21:2356-2361.

10. Custodis F, Baumhakel M, Schlimmer N, et al. Heart rate reduction by ivabradine reduces oxidative stress, improves endothelial function, and prevents atherosclerosis in apolipoprotein E-deficient mice. Circulation 2008;117:2377-2387.

11. Haddad P, Dussault S, Groleau J, Turgeon J, Maingrette F, Rivard A. Nox2-derived reactive oxygen species contribute to hypercholesterolemia-induced inhibition of neovascularization: effects on endothelial progenitor 
cells and mature endothelial cells. Atherosclerosis 2011;217:340-349.

12. Lam YT. Critical roles of reactive oxygen species in age-related impairment in ischemia-induced neovascularization by regulating stem and progenitor cell function. Oxid Med Cell Longev 2015;2015:7095901.

13. Drummond GR, Sobey CG. Endothelial NADPH oxidases: which NOX to target in vascular disease? Trends Endocrinol Metab 2014;25:452-463.

14. Jiang F, Zhang Y, Dusting GJ. NADPH oxidase-mediated redox signaling: roles in cellular stress response, stress tolerance, and tissue repair. Pharmacol Rev 2011;63:218242.

15. Ushio-Fukai M, Urao N. Novel role of NADPH oxidase in angiogenesis and stem/progenitor cell function. Antioxid Redox Signal 2009;11:2517-2533.

16. Fenyo IM, Florea IC, Raicu M, Manea A. Tyrphostin AG490 reduces NAPDH oxidase activity and expression in the aorta of hypercholesterolemic apolipoprotein E-deficient mice. Vascul Pharmacol 2011;54:100-106.

17. Du F, Zhou J, Gong R, et al. Endothelial progenitor cells in atherosclerosis. Front Biosci (Landmark Ed) 2012;17:2327-2349.

18. Madonna R, De Caterina R. Circulating endothelial progenitor cells: do they live up to their name? Vascul Pharmacol 2015;67-69:2-5.

19. Huang Q, Qin L, Dai S, et al. AIP1 suppresses atherosclerosis by limiting hyperlipidemia-induced inflammation and vascular endothelial dysfunction. Arterioscler Thromb Vasc Biol 2013;33:795-804.

20. Yoder MC. Human endothelial progenitor cells. Cold Spring Harb Perspect Med 2012;2:a006692.

21. Kawahara T, Lambeth JD. Molecular evolution of Phoxrelated regulatory subunits for NADPH oxidase enzymes.
BMC Evol Biol 2007;7:178

22. Lassegue B, San Martin A, Griendling KK. Biochemistry, physiology, and pathophysiology of NADPH oxidases in the cardiovascular system. Circ Res 2012;110:1364-1390.

23. Chen DD, Dong YG, Yuan H, Chen AF. Endothelin 1 activation of endothelin A receptor/NADPH oxidase pathway and diminished antioxidants critically contribute to endothelial progenitor cell reduction and dysfunction in salt-sensitive hypertension. Hypertension 2012;59:1037-1043.

24. Lou Z, Ren KD, Tan B, et al. Salviaolate protects rat brain from ischemia-reperfusion injury through inhibition of NADPH oxidase. Planta Med 2015;81:1361-1369.

25. Shao B, Bayraktutan U. Hyperglycaemia promotes human brain microvascular endothelial cell apoptosis via induction of protein kinase C- $\beta \mathrm{I}$ and prooxidant enzyme NADPH oxidase. Redox Biol 2014;2:694-701.

26. Urao N, Inomata H, Razvi M, et al. Role of NOX2-based NADPH oxidase in bone marrow and progenitor cell function involved in neovascularization induced by hindlimb ischemia. Circ Res 2008;103:212-220.

27. Zhang YS, Liu B, Luo XJ, et al. Nuclear cardiac myosin light chain 2 modulates NADPH oxidase 2 expression in myocardium: a novel function beyond muscle contraction. Basic Res Cardiol 2015;110:38.

28. Zhu K, Kakehi T, Matsumoto M, et al. NADPH oxidase NOXı is involved in activation of protein kinase $\mathrm{C}$ and premature senescence in early stage diabetic kidney. Free Radic Biol Med 2015;83:21-30.

29. Liu B, Li T, Peng JJ, et al. Non-muscle myosin light chain promotes endothelial progenitor cells senescence and dysfunction in pulmonary hypertensive rats through up-regulation of NADPH oxidase. Eur J Pharmacol 2016;775:67-77. 